\title{
Resectable Pancreatic Acinar Cell Carcinoma
}

National Cancer Institute

\section{Source}

National Cancer Institute. Resectable Pancreatic Acinar Cell Carcinoma. NCI Thesaurus.

Code C156907.

Pancreatic acinar cell carcinoma that is amenable to surgical resection. 